\title{
Providing Explainable Race-Time Predictions and Training Plan Recommendations to Marathon Runners
}

\author{
Ciara Feely \\ ciara.feely@ucdconnect.ie \\ Aonghus Lawlor \\ Insight Center for Data Analytics \\ aonghus.lawlor@ucd.ie
}

The SFI Center for Research Training in Machine Learning

\author{
Brian Caulfield \\ Insight Center for Data Analytics \\ b.caulfield@ucd.ie \\ Barry Smyth \\ Insight Center for Data Analytics \\ barry.smyth@ucd.ie
}

\begin{abstract}
Millions of people participate in marathon events every year, typically devoting at least $12-16$ weeks to building their endurance and fitness so that they can safely complete these gruelling $42.2 \mathrm{~km}$ races. Most runners follow a training plan that is tailored to their expected finish-time (e.g. sub-4 hours or 4-5 hours), and these plans will prescribe a complex mixture of training sessions to help them achieve these times. However, such plans cannot adapt to the individual needs (fitness levels, changing goals, personal preferences) of runners, providing only broad training guidance rather than more personalised support. The development of wearable sensors and mobile fitness applications facilitates the collection of a large amount of training data from runners. In this paper, we propose a recommender system that utilizes such training data to deliver more personalised training advice to runners, using ideas from casebased reasoning to reuse and adapt the training habits of similar runners. Explainability plays a significant role in this type of system, and we also describe how the predictions and recommendation advice can be presented to runners. An initial off-line evaluation is presented based on a large-scale, real-world dataset.
\end{abstract}

\section{CCS CONCEPTS}

- Computing methodologies $\rightarrow$ Machine learning algorithms; Feature selection; • Applied computing $\rightarrow$ Health informatics.

\section{KEYWORDS}

recommender systems, case-based reasoning, marathon running, race-time prediction, training plan recommendation

ACM Reference Format:

Ciara Feely, Brian Caulfield, Aonghus Lawlor, and Barry Smyth. 2020. Providing Explainable Race-Time Predictions and Training Plan Recommendations to Marathon Runners. In Fourteenth ACM Conference on Recommender Systems (RecSys '20), September 22-26, 2020, Virtual Event, Brazil. ACM, New York, NY, USA, 6 pages. https://doi.org/10.1145/3383313.3412220

\section{INTRODUCTION}

The number of people participating in endurance events, such as marathons, is increasing yearly. While professional athletes may

This work is licensed under a Creative Commons Attribution International 4.0 License. RecSys '20, September 22-26, 2020, Virtual Event, Brazil

(C) 2020 Copyright held by the owner/author(s).

ACM ISBN 978-1-4503-7583-2/20/09.

https://doi.org/10.1145/3383313.3412220 have a team of coaches and personal trainers to support their endeavours, recreational runners often have to fend for themselves. Most recreational runners train by following reasonably generic advice that is, at best, tailored to their projected or target finishtime. The recent availability of wearable sensors and mobile fitness applications promises to re-balance this state of affairs by facilitating the provision of tailored training advice [Boratto et al. 2018; Cau et al. 2019; Monteiro-Guerra et al. 2019; Mulas et al. 2013], personalised motivational supports [Boratto et al. 2017; Buttussi et al. 2006; Hosseinpour and Terlutter 2019; Mulas et al. 2011; Pilloni et al. 2018], sophisticated performance analysis and prediction [Bartolucci and Murphy 2015; Keogh et al. 2019], and even in-race guidance [Berndsen et al. 2019]. In this work, we build on these ideas by using raw training data to support marathon runners in two important ways: (1) by predicting their projected marathon time at different points in their training; and (2) by providing tailored training recommendations based on their recent training and their current performance goals.

Race-time prediction is an important task as many runners will calibrate their training based on their estimated finish time. While this task has been explored previously, the approaches have typically used data from the entire training process or past race-times [Bartolucci and Murphy 2015; Claudino et al. 2019; Doherty et al. 2019; Keogh et al. 2019] to generate predictions just before race-day. For example, the recent work of [Smyth and Cunningham 2017a,b, 2018a,b] was able to generate accurate finish-time predictions but only if the runner had completed at least one recent marathon, and as such it was not suitable for first-timers. Our focus is on making in-training predictions, which can be generated for novices and experts alike, based on their recent training sessions. These predictions not only serve to help the runner calibrate their race-day goals but also help them to better understand the effectiveness of their training.

Moreover, if their expectations change during training - if things are going well they may want to opt for a more challenging goal or vice versa if their recent training has been too challenging or if they have suffered a disruption (e.g. an injury or period of illness) then they can request a revised training plan based on their recent training and their new goals. While the promise of personalised training recommendations has long been cited as an important goal for these types of system [Monteiro-Guerra et al. 2019; Schneider 2017; Zahran et al. 2019] progress has so far been limited, notwithstanding some noteworthy early attempts such as [Fister Jr and Fister 2017]. This is, in part at least, because generating personalised training plans ordinarily requires a deep domain model to use as the basis for decision making and recommendation. In this work, 
we propose an alternative approach, by using case-based reasoning methods [Smyth 2007] to suggest training adjustments based on the training of similar runners in the past, thereby harnessing their training choices but without the need for a deep domain model.

In this work, we extend the recent work of [Feely et al. 2020]. Our main technical contribution is two-fold. First, we use feature selection techniques to identify important, changing indicators of training performance as training unfolds; we also show how we can use past performance predictions as additional features for future prediction setups. Second, we demonstrate how this new representational approach facilitates a more transparent and explainable form of training recommendation for the end-user. The work is evaluated using a real-world dataset of over 21,000 marathoners.

\section{A CASE-BASED APPROACH TO PERFORMANCE PREDICTION AND TRAINING RECOMMENDATION}

We describe our case-based approach to prediction and recommendation and why this approach was chosen in the context of marathon training.

\subsection{Why a Case-Based Approach?}

Most marathon training programmes involve 12-16 weeks of training, usually with 3-6 sessions per week depending on ability and goals. They involve complex sequences of different types of sessions, designed with different goals for different points in a training cycle (e.g. building an endurance base vs. speed and strength). As such, the ability to algorithmically generate and recommend a complex multi-faceted training programme requires a deep understanding of human physiology, running mechanics, and the particular mental and physical challenges of the marathon event.

One of the advantages of case-based reasoning $(\mathrm{CBR})$, is that it can make up for a lack of a deep domain model: a CBR system will solve new problems by reusing and adapting the solutions of similar past problems, and there is a long history of using CBR in applications where deep domain models are either not available or are not feasible to use, from legal reasoning [Ashley 1991] to manufacturing [Hinkle and Toomey 1995] to scheduling [Cunningham and Smyth 1997]. In the context of marathon training, this suggests that it may be possible to deliver more targeted training advice to a runner by harnessing the training plans used by similar runners at a similar point in their training cycle. For example, if a runner who has been training for and is on-track to achieve a 4-hour finish, decides to target a 3:50 finish-time, then next week's training can be recommended from the plans of similar runners who have achieved this faster time in the past. Note that the training plan recommended based on similar runners is considered appropriate since the runners achieved the target runner's goal finish-time by employing that specific training. This is a powerful idea. It means that a coherent week of training can be recommended without the need for a deep domain model. This is extremely beneficial since marathon training is complex and success in the sport is multifactorial, making the domain model difficult to implement. The essence of best practice for marathon running is contained in the training plans which is what is utilized in the case-base reasoning system. Additionally, case-based reasoning is preferable to other forms of recommender systems techniques since it can harness the type of data that is routinely collected by smart-devices and doesn't require explicit ratings or additional data from the runners.

\section{$2.2 \quad$ A Feature-Based Representation of Training Session Data}

In this work, each individual training session/activity is comprised of a sequence of timestamps and elevations $(\mathrm{m})$ every $100 \mathrm{~m}$ for a particular training run. The timing information can be used to calculate basic pacing information (minutes per $\mathrm{km}$ ) as in the work of [Feely et al. 2020]. Then, the training plan for runner $r$, can be represented as a time-ordered sequence of activities; where each activity $A_{i}(r)$ indicates the number of days before the race, $d$, the activity's pacing, $P$, and elevation data, $E$.

$$
T(r)=\left\{A_{1}(r), A_{2}(r), \ldots, A_{n}(r)\right\}
$$

$$
A_{i}(r)=(d, P, E)
$$

\subsection{Case Representation}

We use this raw training data to generate a training case by aggregating the activities on a week by week basis. Thus, the case $C(r, w)$ represents $r$ 's training in week $w$ and it is made up of three basic components:

(1) $F(r, w)$ is a set of features which summarise $r$ 's training in week $w$.

(2) $M T(r)$ is the the marathon time achieved by $r$ in their target race.

(3) $C(r, w-1)$ is a pointer to $r$ 's next week of training

Note that $w$ is weeks from the race so week 6 is 6 weeks from the race. Hence $w-1$ is closer to the race than $w$ and refers to the training week after $w$. In case-based reasoning parlance, $F(r, w)$ corresponds to the problem description and will be used to determine case similarity, when comparing cases to some new target problem, that is a query runner looking for finish-time predictions and/or training recommendations. And $M T(r)$ and $C(r, w-1)$ are case solutions, which will be adapted for finish-time prediction or training recommendation if a case is reused for a query runner.

The descriptive features of a case, $F(r, w)$ are critical to the success of any CBR solution: using the right features makes it possible to identify meaningful similarities between cases facilitating relevant retrieval and reuse. Using the wrong features will facilitate incorrect reminding: irrelevant cases will be retrieved, and their solutions will not be appropriate to the current target problem. In this work, the basic features that make up $F(r, w)$ include: training frequency (number of sessions and number of training days in the week); total weekly distance; the distance of the longest activity; the number of activities at different distances $-<5 \mathrm{~km}, 5-10 \mathrm{~km}$, $10-20 \mathrm{~km}$ and $>20 \mathrm{~km}$; the mean and standard deviation of weekly pace; the fastest $1 \mathrm{~km}, 5 \mathrm{~km}$, and $10 \mathrm{~km}$ paces; the pace of the longest activity; the average pacing split (the difference between first and second half pacing in a session). These are all common features that runners will pay heed to during their training. In addition, $F(r, w)$ also includes cumulative pacing values, corresponding to the above 
pacing features. This feature set is an extension of previous work [Feely et al. 2020], with some new features.

\subsection{Task 1: Race-Time Prediction}

The purpose of this task is to generate an estimate for a query runner $r$ 's marathon time $w$ weeks from race-day. This will help $r$ to set and maintain realistic race expectations but it also helps them to track how well their training is progressing relative to their current goal-time; if their predicted race-time is much slower than their goal-time, then it may be time to reassess their goal or adjust their training, for example. In what follows, we will describe three alternatives to generating such a finish-time prediction for $r$ at week $w$.

2.4.1 Baseline (B).. The simplest approach is to use $F(r, w)$ in $C(r, w)$ as a query to select the $k$ most similar cases $\left(c_{1}^{\prime}, \ldots, c_{k}^{\prime}\right)$ based on the similarity between $F(r, w)$ in the query, and the corresponding $F(r, w)$ in cases for the corresponding training week and gender. We use a standard Euclidean distance metric to compute this similarity. Then, we compute the similarity-weighted mean of the marathon times for the $k$ most similar cases to use as the predicted time for $r$. This baseline is similar to the single-week approach used by [Feely et al. 2020].

2.4.2 Feature Selection (FS).. One of the shortcomings of the baseline is that oftentimes prediction accuracy can decrease in higher dimensional spaces [Bellman et al. 1957] and the baseline uses every feature to determine similarity even though some features (e.g. longest run distance or fastest $10 \mathrm{~km}$ pace or number of sessions per week) may be more or less relevant during different weeks of a training programme. Accordingly, in this variant we use a forward, sequential feature selection procedure [Hocking 1976] to select a subset of relevant features for a given week of training. In addition to improving prediction accuracy, this approach has the potential to improve the interpretability or explainability of predictions and recommendations, since they are based on a smaller set of features that can be ranked by importance.

2.4.3 Multi-week Features (MW).. Our final variation is a response to the criticism that the approach so far is limited to the use of information about a single week of training; that is, the prediction for week $w-1$ is based on the training in week $w$. The work of [Feely et al. 2020] addressed this by using an ensemble approach to combine the previous 4 weeks of training, but this made predictions and recommendations even harder to explain, because now they were based on multiple weeks of training, each with its own complex set of features. In this work, we propose an alternative by including the finish-time predictions from previous weeks as additional features for the current week, as per Equation 3. Then, prediction proceeds using the feature selection variant with the expanded feature sets $F^{\prime}(r, w)$

$$
F^{\prime}(r, w)=F(r, w) \cup\left\{M T\left(r, w^{\prime}\right) \forall w^{\prime}>w\right\}
$$

\subsection{Task 2: Training Plan Recommendation}

The goal of the training plan recommendation task is to recommend next week's training to $r$ based on their current week of training and a modified marathon finish-time, $M T * \delta$. For example, if training has been going well then a runner may wish to explore the training requirements associated with a finish-time that is $2 \%$ faster than their current goal time $M T$, hence the adjusted goal-time is $M T * 0.98$. Or if training has been proceeding poorly, then they may need to adjust their expectations and seek training advice for a slower finish-time $(\delta>1)$.

This time we use a two-step CBR process. First we select the $k$ nearest neighbours $(n n)$ to the query case, based on $F^{\prime}(r, w)$, from the subset of week $w$ cases that are within 5 minutes less than (greater than) $r$ 's modified finish-time if this time is less than (greater than) their predicted time; these $k$ nearest neighbours have had a similar week $w$ to our query runner but their subsequent marathon times are close to the revised goal-time, and as such we can expect their next training week to be broadly suitable for the query runner. Rather than recommend one of these training weeks to the query runner, we instead produce a new pseudo case from these $k$ similar cases. The features of this pseudo case, $\overline{F^{\prime}(n n, w)}$, are the average of the features of the nearest neighbours and these are used to select a single best nearest neighbour to use as the basis for next week's training sessions. If instead we had selected the single-best neighbour in step one then we would run the risk of recommending an unrepresentative next week of training if, for example, the nearest runner had a down-week, as is often the case with training programmes.

\section{EVALUATION}

\subsection{Data \& Methodology}

This evaluation uses an anonymised, real-world dataset of 1.5 million training activities for 21,840 runners of the Dublin, London, and New York marathons during the period from 2014-2017; The dataset was made available to the authors through a data-sharing agreement with the popular mobile fitness application, Strava. The data collected by Strava consists of timing and elevation data for the individual training sessions completed by users as collected by off-the-shelf wearable sensors, smartwatches, and smartphones, which are now commonplace. For the purpose of this evaluation we focused on the 4,810 female runners with finish-times between 3 and 5 hours and the 15,120 male runners with finish-times less than 5 hours since there were few slower runners, and few quicker female runners that made accurate race-time predictions and training plan recommendations difficult.

The race-time prediction evaluation used a standard 10-fold cross-validation approach, using $10 \%$ of cases as test problems and the remaining $90 \%$ as cases to use for prediction, comparing the predicted finish-time to the actual finish-time to compute an average RMSE (measured in minutes); in what follows we describe the RMSE results for $k=15$, which was found to be the point of error stabilisation for the three prediction variants $(B, F S$, and $M W)$.

Recommending training plans represents a more challenging evaluation. Ideally it requires a live-user trial, with real runners responding to the advice. However, this is beyond the scope of this short paper. As an alternative we provide a preliminary proof-ofconcept style evaluation that attempts to determine the reasonableness of the recommendations. 


\subsection{Prediction Error}

A key benefit of the approach described is that it accommodates race-time predictions throughout a training programme and the key questions for evaluation are: (a) how accurate are these predictions; and (b) how do they evolve as training progresses. The predictions should be as accurate as possible but accuracy should also improve as training progresses.

To test this, in Figure 1 we present the RMSE for predictions made during each week of training for the different algorithms. In all cases, prediction error decreases as training progresses and from week 13 the $M W$ approach consistently beats all others; as training progresses the differences in RMSE between the $M W$ approach and the others becomes statistically significant based on a onesided $t$ test with $p<0.01$. Since the purpose of this work is to extend [Feely et al. 2020] by finding an accurate prediction model that acts more like a single week model, it is worth noting that the $M W$ approach in this work performs significantly better than the single week approach in the previous work, while performing as well as the best ensemble approach presented. Note that there is a small increase in error for all but the $M W$ approach in the last two weeks of training. This is likely due to the taper effect in marathon training, where runners are advised to significantly reduce their training intensity in the 2-3 weeks before race-day, for recovery. This clearly has a negative impact on finish-time predictions, except for the $M W$ alternative, presumably due to the fact that it retains information from non-taper weeks. Another benefit of the approaches described is that they facilitate a form of explanation so that the target runner can better understand the basis for their finish-time prediction. For example, the system can provide the following output accompanying a predicted race-time of 275 minutes: "This race-time was calculated based on 15 runners that had similar training to you and achieved race-times in the range 260-290 minutes." The same upper and lower values as well as average values for important training features can also be given.

\subsection{On the Reasonableness of Recommendations}

As discussed, it was not feasible to conduct a live-user trial of training recommendations, although this is planned for the future. A recommended training week would ideally resemble the prototype in Figure 2a, based on a runner's current training and their revised goal-time: in this case, for a 3:45 marathon goal-time the runner is recommended a 5-session week consisting of easy, recovery runs and more challenging interval and temp sessions, plus a weekend long run, each with a prescribed duration and pace. As an alternative to a live-user trial we consider an offline evaluation of the reasonableness of recommendations by comparing the recommended training weeks when a runner requests a slightly slower or faster time than their current predicted time. We do this by comparing the average pace of recommended next week of training to the average pace of their default plan for next week. If their new goal-time is faster, it is reasonable to expect that their recommended next training week should be faster than their default week, and vice versa.

Figures $2 \mathrm{~b}$ and $2 \mathrm{c}$ show the results of this for men and women based on different values of $\delta$ at different points in training: faster
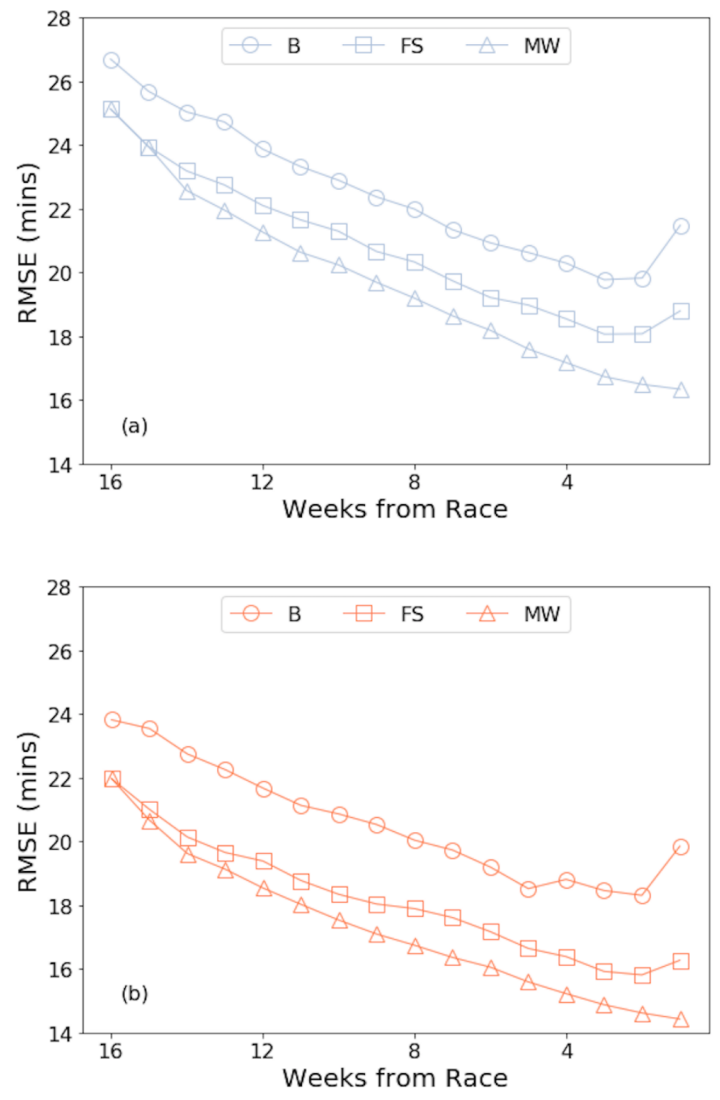

Figure 1: The prediction error (RMSE in minutes) by training week for (a) men and (b) women using the three single-week variants: baseline, feature selection, and multi-week feature

goal-time revisions $(\delta<1)$ result in faster training recommendations (difference $<0$ because pacing is the inverse of speed and so lower pacing values mean faster running) and how slower goaltime revisions lead to slower training recommendations. We find a similar result when we look at total weekly distance. This is expected from training recommendations but, of course, it doesn't necessarily mean that runners will find the specifics of the proposed training weeks to be acceptable, hence the need for a live user trial and further evaluation work.

\section{CONCLUSIONS}

This short paper builds on recent research [Berndsen et al. 2019; Smyth 2019; Smyth and Cunningham 2017b, 2018a] on the novel application of recommender systems to marathon running. We describe how to use the raw training data routinely collected by training apps such as Strava and RunKeeper to provide supports for runners during their training. In particular, we evaluated several variants for making finish-time predictions at any point during training and considered the related task of recommending revised training plans if a runner's goal changes during training. Initial 


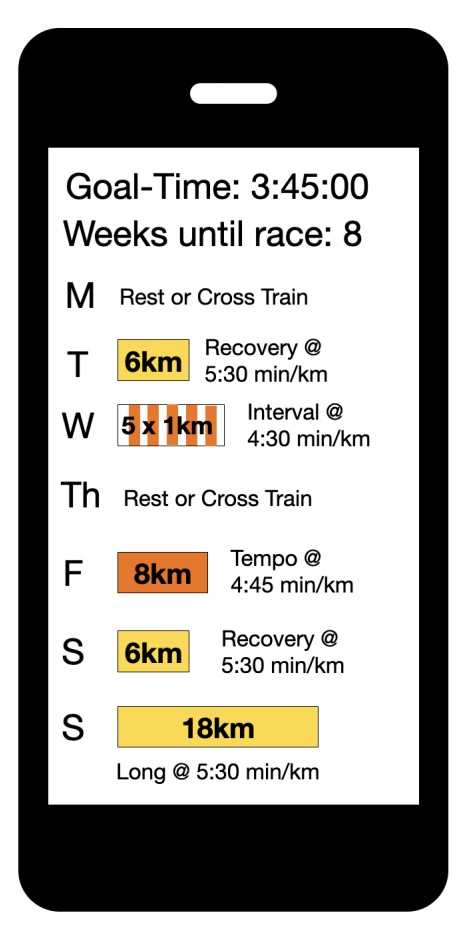

(a)
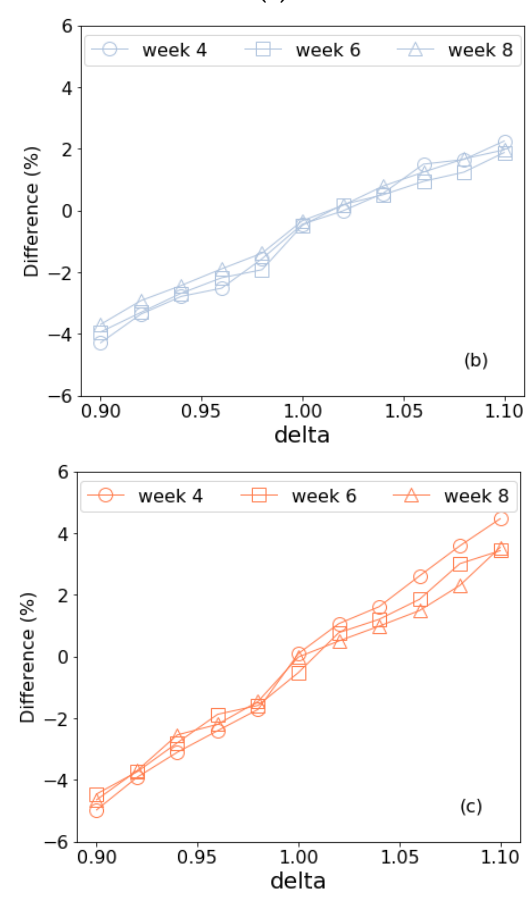

Figure 2: (a): A sample training plan recommendation for a runner with a goal-time of 3:45, 8 weeks from race-day. (b): The percentage difference in mean weekly pace $(\mathrm{mins} / \mathrm{km})$ for training plans based on adjusted goal-times $M T * \delta$ for men and for women (c) during training weeks 4,6 , and 8 . Note: $\delta<1$ indicates that the goal-time is $(1-\delta) \%$ faster than the runner's current predicted time. offline evaluation results using a large dataset of $1.5 \mathrm{M}$ training activities are positive: increasingly accurate predictions are produced as training progresses, with some evidence that the revised training plans recommended to runners are reasonable - establishing the need for a live-user trial to follow. Lastly, exploring the related tasks of training session classification and injury prediction would complement the recommendation of suitable training plans, as would having the plans evaluated by real marathon coaches.

\section{ACKNOWLEDGMENTS}

Supported by Science Foundation Ireland (SFI) through the Insight Centre for Data Analytics (12/RC/2289_P2) and the SFI Centre for Research Training in Machine Learning (18/CRT/6183).

\section{REFERENCES}

Kevin D Ashley. 1991. Modeling legal arguments: Reasoning with cases and hypotheticals. MIT press.

Francesco Bartolucci and Thomas Brendan Murphy. 2015. A finite mixture latent trajectory model for modeling ultrarunners' behavior in a 24-hour race. fournal of Quantitative Analysis in Sports 11, 4 (2015), 193-203. https://doi.org/10.1515/jqas2014-0060

R. Bellman, Rand Corporation, and Karreman Mathematics Research Collection. 1957. Dynamic Programming. Princeton University Press. https://books.google.it/books? id=wdtoPwAACAAJ

Jakim Berndsen, Barry Smyth, and Aonghus Lawlor. 2019. Pace my race: recommendations for marathon running. In Proceedings of the 13th ACM Conference on Recommender Systems. ACM, 246-250.

Ludovico Boratto, Salvatore Carta, Gianni Fenu, Matteo Manca, Fabrizio Mulas, and Paolo Pilloni. 2017. The role of social interaction on users motivation to exercise: A persuasive web framework to enhance the self-management of a healthy lifestyle. Pervasive and Mobile Computing 36 (2017), 98-114. https://doi.org/10.1016/j.pmcj. 2016.08.009

Ludovico Boratto, Salvatore Carta, Walid Iguider, Fabrizio Mulas, and Paolo Pilloni. 2018. Predicting Workout Quality to Help Coaches Support Sportspeople. In Proceedings of the 3rd International Workshop on Health Recommender Systems, HealthRecSys 2018, co-located with the 12th ACM Conference on Recommender Systems (ACM RecSys 2018), Vancouver, BC, Canada, October 6, 2018. 8-12. http://ceurws.org/Vol-2216/healthRecSys18_paper_2.pdf

Fabio Buttussi, Luca Chittaro, and Daniele Nadalutti. 2006. Bringing Mobile Guides and Fitness Activities Together: A Solution Based on an Embodied Virtual Trainer. In Proceedings of the 8th Conference on Human-computer Interaction with Mobile Devices and Services (Helsinki, Finland) (MobileHCI '06). ACM, New York, NY, USA, 29-36. https://doi.org/10.1145/1152215.1152222

Federico Maria Cau, Mattia Samuel Mancosu, Fabrizio Mulas, Paolo Pilloni, and Lucio Davide Spano. 2019. An intelligent interface for supporting coaches in providing running feedback. In Proceedings of the 13th Biannual Conference of the Italian SIGCHI Chapter: Designing the next interaction, CHItaly 2019, adova, Italy, September 23-25, 2019. 6:1-6:5. https://doi.org/10.1145/3351995.3352039

João Gustavo Claudino, Daniel de Oliveira Capanema, Thiago Vieira de Souza, Julio Cerca Serrão, Adriano C. Machado Pereira, and George P. Nassis. 2019. Current Approaches to the Use of Artificial Intelligence for Injury Risk Assessment and Performance Prediction in Team Sports: A Systematic Review. Sports Medicine Open 5, 1 (July 2019), 28. https://doi.org/10.1186/s40798-019-0202-3

Pádraig Cunningham and Barry Smyth. 1997. Case-based reasoning in scheduling: reusing solution components. International fournal of Production Research 35, 11 (1997), 2947-2962.

Cailbhe Doherty, Alison Keogh, James Davenport, Aonghus Lawlor, Barry Smyth, and Brian Caulfield. 2019. An evaluation of the training determinants of marathon performance: A meta-analysis with meta-regression. Fournal of science and medicine in sport (2019).

Ciara Feely, Brian Caulfield, Aonghus Lawlor, and Barry Smyth. 2020. Using CaseBased Reasoning to Predict Marathon Performance and Recommend Tailored Training Plans. In Case-Based Reasoning Research and Development - 28th International Conference, ICCBR 2020, Salamanca, Spain, June 8-12, 2020, Proceedings.

Iztok Fister Jr and Iztok Fister. 2017. Generating the training plans based on existing sports activities using swarm intelligence. In Nature-Inspired Computing and Optimization. Springer, 79-94.

David Hinkle and Christopher Toomey. 1995. Applying case-based reasoning to manufacturing. AI magazine 16, 1 (1995), 65-65.

R. R. Hocking. 1976. A Biometrics Invited Paper. The Analysis and Selection of Variables in Linear Regression. Biometrics 32, 1 (1976), 1-49. http://www.jstor.org/stable/ 2529336 
Masoumeh Hosseinpour and Ralf Terlutter. 2019. Your Personal Motivator is with You A Systematic Review of Mobile Phone Applications Aiming at Increasing Physical Activity. Sports Medicine 49, 9 (01 Sep 2019), 1425-1447. https://doi.org/10.1007/ s40279-019-01128-3

Alison Keogh, Barry Smyth, Brian Caulfield, Aonghus Lawlor, Jakim Berndsen, and Cailbhe Doherty. 2019. Prediction Equations for Marathon Performance: A Systematic Review. International fournal of Sports Physiology and Performance 14, 9 (2019), 1159-1169.

Francisco Miguel Monteiro-Guerra, Octavio Rivera-Romero, Luis Fernandez Luque, and Brian Caulfield. 2019. Personalization in Real-Time Physical Activity Coaching using Mobile Applications: A Scoping Review. IEEE journal of biomedical and health informatics (2019)

Fabrizio Mulas, Salvatore Carta, Paolo Pilloni, and Matteo Manca. 2011. Everywhere run: a virtual personal trainer for supporting people in their running activity. In Proceedings of the 8th International Conference on Advances in Computer Entertainment Technology, ACE 2011, Lisbon, Portugal, November 8-11, 2011. 70. https://doi.org/10.1145/2071423.2071510

Fabrizio Mulas, Paolo Pilloni, Matteo Manca, Ludovico Boratto, and Salvatore Carta. 2013. Using new communication technologies and social media interaction to improve the motivation of users to exercise. In Second International Conference on Future Generation Communication Technologies (FGCT 2013), London, United Kingdom, November 12-14, 2013. 87-92. https://doi.org/10.1109/FGCT 2013.6767189

Paolo Pilloni, Luca Piras, Salvatore Carta, Gianni Fenu, Fabrizio Mulas, and Ludovico Boratto. 2018. Recommender System Lets Coaches Identify and Help Athletes Who Begin Losing Motivation. IEEE Computer 51, 3 (2018), 36-42. https://doi.org/10 1109/MC.2018.1731060

Hanna Schneider. 2017. Adapting at Run-time: Exploring the Design Space of Personalized Fitness Coaches. In Proceedings of the 22Nd International Conference on
Intelligent User Interfaces Companion (Limassol, Cyprus) (IUI '17 Companion). ACM, New York, NY, USA, 173-176. https://doi.org/10.1145/3030024.3038280

Barry Smyth. 2007. Case-Based Recommendation. In The Adaptive Web, Methods and Strategies of Web Personalization. 342-376. https://doi.org/10.1007/978-3-54072079-9_11

Barry Smyth. 2019. Recommender Systems: A Healthy Obsession. In Proceedings of the AAAI Conference on Artificial Intelligence, Vol. 33. 9790-9794.

Barry Smyth and Pádraig Cunningham. 2017a. A Novel Recommender System for Helping Marathoners to Achieve a New Personal-Best. In Proceedings of the Eleventh ACM Conference on Recommender Systems, RecSys 2017, Como, Italy, August 27-31, 2017. 116-120. https://doi.org/10.1145/3109859.3109874

Barry Smyth and Pádraig Cunningham. 2017b. Running with Cases: A CBR Approach to Running Your Best Marathon. In Case-Based Reasoning Research and Development - 25th International Conference, ICCBR 2017, Trondheim, Norway, fune 26-28, 2017, Proceedings. 360-374. https://doi.org/10.1007/978-3-319-61030-6 25

Barry Smyth and Pádraig Cunningham. 2018a. An Analysis of Case Representations for Marathon Race Prediction and Planning. In Case-Based Reasoning Research and Development - 26th International Conference, ICCBR 2018, Stockholm, Sweden, Fuly 9-12, 2018, Proceedings. 369-384. https://doi.org/10.1007/978-3-030-01081-2_25

Barry Smyth and Padraig Cunningham. 2018b. Marathon Race Planning: A CaseBased Reasoning Approach. In Proceedings of the Twenty-Seventh International foint Conference on Artificial Intelligence, IfCAI 2018, fuly 13-19, 2018, Stockholm, Sweden. 5364-5368. https://doi.org/10.24963/ijcai.2018/754

Laila Zahran, Mohammed El-Beltagy, and Mohamed Saleh. 2019. A Conceptual Framework for the Generation of Adaptive Training Plans in Sports Coaching. In International Conference on Advanced Intelligent Systems and Informatics. Springer, 673-684. 